\title{
A CINESIOTERAPIA NO TRATAMENTO DA FIBROMIALGIA: REVISÃO BIBLIOGRÁFICA.
}

KINESIOTHERAPY IN THE TREATMENT OF FIBROMYALGIA:

LITERATURE REVIEW.

\section{Jordana de Faria Arantes}

Docente do curso de Fisioterapia da Faculdade Evangélica de Ceres - AEE. Ceres-Go, Brasil. Mestre em Atenção à Saúde.

E-mail: jordana_arantes@ @otmail.com

\section{Renata Sousa Nunes}

Docente do curso de Fisioterapia da Faculdade Evangélica de Ceres - AEE. Ceres- Go, Brasil. Especialista em Docência Universitária.

E-mail: renatafisio8@hotmail.com

\section{Anna Luiza Teixeira}

Acadêmica do curso de Fisioterapia da Faculdade Evangélica de Ceres - AEE. Ceres - Go, Brasil.

E-mail: anninhalt@live.com

\section{Davilla Gonçalves de Souza}

Acadêmica do curso de Fisioterapia da Faculdade Evangélica de Ceres - AEE. Ceres - GO, Brasil.

E-mail: davillag.souza@gmail.com

\section{Waghner Frank Ribeiro Pereira}

Acadêmico do curso de Fisioterapia da Faculdade Evangélica de Ceres, Ceres - GO.

E-mail: waghner1@ @otmail.com

\section{RESUMO}


Introdução: A fibromialgia é uma síndrome reumática de etiologia desconhecida, que acomete predominantemente mulheres, caracterizada por dor musculoesquelética difusa e crônica, sítios anatômicos específicos dolorosos à palpação (tender points). A fisioterapia tem sido indicada no tratamento da FM, pois possibilita a análise e a intervenção na mobilidade humana, reintegrando o paciente ao meio onde vive e, promovendo assim, uma melhora na qualidade de vida. Objetivo: Revisar os estudos da literatura, a fim de verificar a eficácia das técnicas de cinesioterapia no tratamento de FM e ampliar as perspectivas sobre a terapêutica dessa patologia. Metodologia: Foram pesquisados estudos publicados entre os anos de 2002 a 2016, em português, na Biblioteca Virtual da Associação Evangélica de Ceres e nas bases de dados eletrônicos Scielo (Scientific Electronic Library Online), Pubmed (United States National Library of Medicine) e Google Acadêmico. Resultados e discussão: Em relação aos exercícios de alongamento muscular, observou-se uma melhora significativa no sono e uma diminuição da rigidez, tendo o alongamento estático, técnica mais comum e segura proporcionado aumento da flexibilidade, ganho de condicionamento físico, diminuição da dor e da fadiga muscular. Exercícios aeróbios por sua vez, trazem benefícios físicos como a diminuição da tensão muscular, disfunção física e dor. Conclusão: O exercício aeróbio tem ganhos mais diversificados do que os exercícios de alongamento. Portanto, se ambas forem executadas juntas ou simultaneamente, podem trazer mais resultados em menor tempo e com maior rendimento.

Palavra-chave: Cinesioterapia. Fibromialgia. Tratamento.

\section{ABSTRACT}

Introduction: Fibromyalgia is a rheumatic syndrome of unknown etiology, which affects women predominantly, characterized by diffuse and chronic musculoskeletal pain, specific anatomical sites painful to palpation (tender points). Physiotherapy has been indicated in the treatment of FM, since it allows the analysis and intervention in human mobility, reintegrating the patient to the environment where he lives and, thus, improving the quality of life. Objective: To review the literature studies in order to verify the efficacy of kinesiotherapy techniques in the treatment of FM and to broaden perspectives on the therapeutics of this pathology. Methodology: Studies published between the years 2002 to 2016, in Portuguese, in the Virtual Library of the Evangelical Association of Ceres and in the electronic databases Scielo (Scientific Electronic Library Online), Pubmed (United States National Library of 
Medicine) and Google Scholar were searched. Results and discussion: The static stretching technique is the most common and safe, with a short application time. It provides increased flexibility, gain in physical conditioning, decreased pain and muscle fatigue. With muscle stretching exercises, significant improvement in sleep and a decrease in stiffness were observed. With regard to physical conditioning, it was found that there were no satisfactory gains. Physical conditioning, on the other hand, does not significantly modify sleep and stiffness. Conclusion: Aerobic exercise has more diversified gains than stretching exercises. Therefore, if both are performed together or simultaneously, they can bring more results in less time and with higher yield.

Keywords: Kinesiotherapy. Fibromyalgia. Treatment.

\section{INTRODUÇÃO}

A fibromialgia (FM) é uma síndrome reumática e de etiologia desconhecida, que acomete predominantemente mulheres. É caracterizada por dor musculoesquelética difusa e crônica, além de sítios anatômicos específicos dolorosos à palpação, chamados de tender points. Frequentemente associa-se à fadiga, ao déficit de memória, cefaléia, distúrbios do sono, rigidez matinal e distúrbios psicológicos, como a ansiedade e depressão (MARQUES et at., 2002).

Os sintomas descritos podem variar de acordo com algumas condições moduladoras. Grau de atividade física (piorando ao realizar atividades que exigem maior intensidade), mudanças no padrão do sono e até mesmo alterações climáticas podem influenciar no quadro clínico do paciente. Por se tratar de uma síndrome complexa, há uma grande necessidade de um bom diálogo com o paciente e se torna indispensável um detalhado exame físico para um diagnóstico correto (MARQUES, A. P. et al., 2015).

A fisioterapia tem sido indicada no tratamento da FM, pois possibilita a análise e a intervenção na mobilidade humana, de forma a capacitar o indivíduo a realizar suas atividades sejam elas ocupacionais, profissionais e de lazer, reintegrando o paciente ao meio onde vive e, promovendo assim, uma melhora na qualidade de vida (MARQUES, A. P. et al., 2015).

Em decorrência da dor induzida ao realizar atividades físicas, grande parte dos indivíduos portadores de FM adotam o sedentarismo como estilo de vida. No tratamento 
fisioterapêutico destaca- se a cinesioterapia, que utiliza o exercício terapêutico para a prevenção e tratamento de doenças (MARQUES, A. P. et al., 2015).

Portanto, o objetivo deste trabalho foi revisar os estudos da literatura, a fim de verificar a eficácia das técnicas de cinesioterapia no tratamento de FM e ampliar as perspectivas sobre a terapêutica dessa patologia.

\section{METODOLOGIA}

O presente artigo constitui-se de uma revisão de literatura narrativa. Foram pesquisados estudos publicados entre os anos de 2002 a 2016, em português, na Biblioteca Virtual da Associação Evangélica de Ceres e nas bases de dados eletrônicos Scielo (Scientific Electronic Library Online), Pubmed (United States National Library of Medicine) e Google Acadêmico.

O levantamento foi realizado com os seguintes descritores: fibromialgia, cinesioterapia, fisioterapia e tratamento. Definiram-se como critérios de inclusão os artigos que abordavam a intervenção da cinesioterapia no tratamento da FM. Foram encontrados 24 artigos, sendo realizada a leitura dos resumos, após, foram excluídos 12 artigos que não se enquadravam nos critérios de inclusão pois apresentavam associação de técnicas de cinesioterapia a outras técnicas fisioterápicas. Sendo assim, foram incluídos na revisão doze artigos e um livro.

\section{RESULTADOS}

Foram encontrados estudos que abordaram as técnicas de exercício físico, exercício aeróbio, exercício de alongamento muscular e hidrocinesioterapia.

Segundo as bibliografias avaliadas, em relação aos exercícios de alongamento muscular, observou-se uma melhora significativa no sono e uma diminuição da rigidez, tendo o alongamento estático, técnica mais comum e segura proporcionando aumento da flexibilidade, ganho de condicionamento físico, diminuição da dor e da fadiga muscular (MARQUES et al., 2007, p. 21). Segundo os autores, o condicionamento físico por sua vez não modifica de maneira significantiva o sono e a rigidez. (BRESSAN et al., 2000, p. 92).

Em relação a prática correta dos exercícios de alongamento, é necessário exercitar os músculos doloridos com exercícios de alongamento e melhorar as condições cardiovasculares 
com exercícios aeróbios, e exercícios físicos de baixa intensidade, que são considerados mais eficazes na diminuição do impacto da doença e na melhoria da qualidade de vida desses pacientes, tanto física, quanto psíquica e social (HECKER et al., 2011, p. 58).

Os exercícios aeróbicos são mais eficientes do que os alongamentos no que tange os aspectos físico e psicológico, sendo o alongamento mais eficaz no tratamento da dor e os exercícios aeróbicos na diminuição gradual da ansiedade (VALIM, 2006, p. 52). Exercícios aeróbios por sua vez, trazem benefícios físicos como a diminuição da tensão muscular, disfunção física e dor (BRESSAN et al., 2000, p. 92).

Foi verificado, ainda, que o treinamento resistido reduz a dor e promove melhoria na qualidade de sono, capacidade funcional, bem-estar geral e controle dos fatores de estresse (REBUTINI et al., 2013, p. 517).

Outra técnica apresentada por autores analisados é a hidrocinesioterapia que consiste na realização de exercícios em água aquecida- em torno de $30^{\circ} \mathrm{C}$ a $34^{\circ} \mathrm{C}$ e proporciona relaxamento muscular, diminuição da dor e rigidez, melhora da circulação sanguínea, além de benefícios ao retorno venoso (HECKER et al., 2011, p. 58). Sendo assim, a hidrocinesioterapia ajuda no combate a dor e melhora da qualidade de vida de pacientes portadores de fibromialgia (JORGE et al., 2016, p. 33).

No âmbito da saúde da mulher, em relação aos sintomas climatéricos, o tratamento cinesioterápico da FM possui efeitos limitantes na capacidade física e na necessidade de descanso em decorrência da cronicidade do processo álgico. Diante disso, evidenciou-se a dificuldade na progressão do tratamento por consequência do início precoce da menopausa, bem como da redução do tempo de exposição ao estrogênio (LISBOA et al., 2015, p. 212, 214).

Os benefícios do tratamento caracterizam- se como uma intervenção de baixo custo financeiro e a correta orientação por parte do fisioterapeuta é indispensável para que os ganhos se perpetuem e, por conseguinte, uma maior independência em relação aos cuidados de saúde seja adquirida (VALIM et al., 2013, p. 539).

\section{DISCUSSÃO}

O tratamento de cinesioterapia promove bem-estar geral devido à liberação de endorfina no sistema nervoso central, através do alongamento muscular e dos exercícios de baixa intensidade (HECKER et al., 2011, p. 61). 
Foram analisadas as seguintes técnicas: exercícios físicos, alongamento muscular, exercícios aeróbios e hidrocinesioterapia.

Segundo Valim e outros (2013, p. 539), o exercício físico possui grande eficácia na redução da dor, no número de pontos dolorosos, melhora na qualidade de vida, humor e outros aspectos psicológicos.

Ao realizar um comparativo entre os exercícios de alongamento e os exercícios aeróbios, foi notável que os exercícios aeróbios atuam com maior eficácia nos sintomas psicológicos, enquanto os exercícios de alongamento atuam de fato na dor, diminuindo assim, os números de tender points e obtendo uma melhora no sono (MATSUTANI; ASSUMPÇÃO; MARQUES, 2012, p. 415).

O exercício aeróbio em relação ao alongamento é mais efetivo na melhora do ganho de componentes físicos e psicológicos. Entretanto, há uma exacerbação dos sintomas no começo de sua aplicação. O tempo necessário para a melhora sintomática pode ser diferente para cada tipo de exercício, porque os benefícios consequentes do alongamento ocorrem em dez semanas e se estabilizam, enquanto a melhora associada ao condicionamento aeróbio é evidente com dez semanas e continua aumentando até vinte semanas. Isso ocorre devido ao tempo necessário para começar a aparecer os efeitos dessa técnica, que a princípio irá proporcionar ganho na aptidão física e consequente melhora clínica (VALIM, 2006, p. 52).

Sendo assim, "os exercícios aeróbios apresentam melhores resultados por obter mais ganhos nos aspectos emocionais, físicos e psicológicos por apresentar mudanças neuroendócrinas necessárias para a melhora do humor" (VALIM, 2006, p. 52). De acordo com Matsutani, Assumpção e Marques (2012, p. 147) "Os exercícios de alongamento são mais eficazes que os exercícios aeróbicos na dor, no número de tender points, no sono e na depressão da FM."

\section{CONCLUSÃO}

A cinesioterapia possui eficácia significativa quando aplicada aos pacientes com FM, pois reduz a dor, rigidez, fadiga muscular, ansiedade, melhora na qualidade do sono, aumenta a flexibilidade, proporciona ganho de condicionamento físico, e melhoras nos aspectos psicológicos. Diversas técnicas trazem benefícios significativos para pessoas acometidas com FM, porém, os exercícios de alongamento são mais eficazes nos aspectos físicos, enquanto os exercícios aeróbios são mais eficazes nos aspectos físicos e psicológicos. É necessário ressaltar que cada técnica possui sua particularidade, benefícios e contra indicações. Sendo 
assim, os exercícios de alongamento e os exercícios aeróbios são mais eficazes no tratamento de FM.

Concluímos assim que o exercício aeróbio tem ganhos mais diversificados do que os exercícios de alongamento. Portanto, se ambas forem executadas juntas ou simultaneamente, podem trazer mais resultados em menor tempo e com maior rendimento.

\section{REFERÊNCIAS}

BATISTA, J. S.; BORGES, A. M.; WIBELINGER, L. M. Tratamento fisioterapêutico na síndrome da dor miofascial e fibromialgia.Rev Dor, v. 25, n. 2, p. 170-4, abr-jun, São Paulo, 2012.

BRESSAN, L. R. et al. Efeitos do alongamento muscular e condicionamento físico no tratamento fisioterápico de pacientes com fibromialgia. Rev Bras Fisioter, 2008.

HECKER, C. D.; MELO, C.; TOMAZONI, S. S.; LOPES MARTINS, R. A. B.; LEAL JUNIOR, E. C. P. Análise dos efeitos da cinesioterapia e da hidrocinesioterapia sobre a qualidade de vida de pacientes com fibromialgia - um ensaio clínico randomizado. Fisioter Mov., v. 24, n. 1, p. 57-64, jan-mar, 2011.

JORGE, M. S. G. et al. Hidrocinesioterapia na dor e na qualidade de vida em indivíduos portadores de fibromialgia. Rev Inspirar, v. 8, n. 1, p. 29-33, 2016.

LISBOA, L. L. et al. Efeito da cinesioterapia na qualidade de vida, função sexual e sintomas climatéricos em mulheres com fibromialgia. Rev. Brasileira de Reumatologia, v.55, n.3, p. 209-215, 2015.

MARQUES, A. P. et al. Exercícios de alongamento ativo em pacientes com fibromialgia: efeito nos sintomas e na qualidade de vida. Fisioterapia e Pesquisa, v. 14, n. 3, p. 18-24, 2007. 
MARQUES, A. P. et al. Fibroamialgia e Fisioterapia: avaliação e tratamento. 2. ed rev. e atual. Barueri, SP: Editora Manole, 2015.

MARQUES, A. P.; MATSUTANI, L. A.; FERREIRA, E. A. G.; MENDONÇA, L. L. F. A Fisioterapia no tratamento de pacientes com fibromialgia: uma revisão da literatura. Rev. Bras Reumatol, v. 42, n. 1, jan/fev, 2002.

MATSUTANI, L. A.; ASSUMPÇÃO, A.; MARQUES, A. P. Exercícios de alongamento muscular e aeróbico no tratamento da fibromialgia: estudo piloto. Fisioter. Mov., v. 25, n. 2, p. 411-418, abr./jun., 2012.

REBUTINI, V. Z. et al. Efeito do treinamento resistido em paciente com fibromialgia: estudo de caso. Motriz, Rio Claro, v. 19, n. 2, p.513-522, abr./jun., 2013.

VALIM, V. Benefícios dos Exercícios Físicos na Fibromialgia.Rev Bras Reumatol, v. 46, n. 1, p. 49-55, jan/fev, 2006.

VALIM, V. et al. Efeitos do exercício físico sobre os níveis séricos de serotonina e seu metabólito na fibromialgia: um estudo piloto randomizado. Rev Bras Reumatol., v. 53, n. 6, p. 538-541, 2013.

WILHELM, J.; SANTOS, R. V. Benefícios da hidrocinesioterapia na fibromialgia: estudo de caso. FisiSenectus, Unochapecó, p.96-103, 2013. 